\title{
A Modern Touch to Farming
}

\author{
Bikash Kumar Behera \\ B.Tech, CSE \\ College of Engineering and \\ Technology, Bhubaneswar
}

\author{
Avishek Das \\ B.Tech, CSE \\ College of Engineering and \\ Technology, Bhubaneswar
}

\author{
Ashis Ku Mishra \\ Lecturer, Department of CSE \\ College of Engineering and \\ Technology, Bhubaneswar
}

\begin{abstract}
The Agricultural productivity in our country does not meet the required output due to the problems like entering of the animals in the farm and unawareness of the moisture level of the farm with the integration of local weather monitoring system. The objective of the project is to protect the plants from threatening animals and to provide the continuous monitoring of the plants. We can integrate IOT with Digital Image processing and wireless sensor network. Existing systems use LASER network that can not isolate the animal interference from human interference. The Proposed System aims at using motion sensors so that it will continuously monitor the output .If some motion is observed in the farm then the webcam is activated and it captures the image. The captured image is compared with the saved image sheet and then it was verified whether the image is of the human or animal. If animal is sensed then the buzzer is on with the message from the GSM module to the owner and email is sent. Moisture level is sensed and then the output is monitored. If the deficiency in water is sensed and then the data from the weather department was analyzed. If there is a possibility of rain during the next 4-5 hours of the operation then the motor for pumping is stopped or else the pump was started and then the irrigation system works. Water flow can also be controlled via the analog input of the soil moisture level. If there is urgent requirement of the irrigation system a reminder via mail is sent to the user for immediate turning on of the dc motor irrigation system remotely via user. A separate website is provided to the user to manually start the irrigation system via the webpage. All the components are interfaced to the Raspberry pi.
\end{abstract}

\section{Keywords}

Raspberry pi,Image processing(Pi-Cam), IOT ,Sensors (Motion/Proximity, Moisture ).

\section{INTRODUCTION}

Agriculture is considered as the basis of life for the human species as it is the main source of food grains and other raw materials. It plays vital role in the growth of country's economy. It also provides large ample employment opportunities to the people. Growth in agricultural sector is necessary for the development of economic condition of the country. Unfortunately, many farmers still use the traditional methods of farming which results in low yielding of crops and fruits. But wherever automation had been implemented and human beings had been replaced by automatic machineries, the yield has been improved. Hence there is need to implement modern science and technology in the agriculture sector for increasing the yield. Most of the papers signifies the use of wireless sensor network which collects the data from different types of sensors and then send it to main server using wireless protocol. The collected data provides the information about different environmental factors which in turns helps to monitor the system. These factors include attack of insects and pests which can be controlled by spraying the crop with proper insecticide and pesticides. Secondly, attack of wild animals and birds when the crop grows up. There is also possibility of thefts when crop is at the stage of harvesting. Even after harvesting, farmers also face problems in storage of harvested crop. So, in order to provide solutions to all such problems, it is necessary to develop integrated system which will take care of all factors affecting the productivity in every stages like; cultivation, harvesting and post harvesting storage. This paper therefore proposes a system which is useful in monitoring the field data as well as controlling the field operations which provides the flexibility. The paper aims at making agriculture smart using automation and IOT technologies. The highlighting features of this paper includes smart GPS based remote controlled robot to perform tasks like; weeding, spraying, moisture sensing, bird and animal scaring, keeping vigilance, etc. Secondly, it includes smart irrigation with smart control based on real time field data. Thirdly, smart warehouse management which includes; temperature maintenance, humidity maintenance and theft detection in the warehouse. Controlling of all these operations will be through any remote smart device or computer connected to Internet and the operations will be performed by interfacing sensors, Wi-Fi or ZigBee modules, camera and actuators with micro-controller and raspberry pi.

\section{LITERATURE SURVEY}

1. Nikesh Gondchawar, Prof. Dr. R. S. Kawitkar et al.[1] have explained that making agriculture smart using automation and IOT technologies is by highlighting the features that includes smart GPS based remote controlled robot to perform tasks like; weeding, spraying, moisture sensing, bird and animal scaring, keeping vigilance, etc. Secondly, it includes smart irrigation with smart control based on real time field data. Thirdly, smart warehouse management which includes; temperature maintenance, humidity maintenance and theft detection in the warehouse. Controlling of all these operations will be through any remote smart device or computer connected to Internet and the operations will be performed by interfacing sensors, Wi-Fi or ZigBee modules, camera and actuators with micro-controller and raspberry.

2. Manoj C Chauhan, Mehul K Vala et al.[2] have explained about the IOT protocols based on which they have connected temperature and humidity sensor, soil moisture sensor, web camera, solenoid valve and speaker with the raspberry pi model . The whole system works on $5 \mathrm{v}$ battery and all data acquisition of field parameters like temperature, humidity, soil moisture and web camera is done using raspberry pi model. Also they have connected solenoid valve and speaker with the raspberry pi model. They have controlled solenoid valve and speaker at the field from remote area by using internet. And, hence the parameters of the field can be controlled and on the field side plant monitoring is done using web camera using 
the whole system the frequency of accusing the field parameters on the web page can be very easily. The frame size and sending data rate for video is also can be very easily by slight changes into the programming. The technological development in Wireless Sensor Networks made it possible to use in monitoring and control of greenhouse parameter in precision agriculture.

3. Y. Kim, R. Evans and W. Iversen et al.[3] have explained that after the research in the agricultural field, researchers found that the yield of agriculture is decreasing day by day. However, use of technology in the field of agriculture plays important role in increasing the production as well as in reducing the extra man power efforts. Some of the research attempts are done for betterment of farmers which provides the systems that use technologies helpful for increasing the agricultural yield. A remote sensing and control irrigation system using distributed wireless sensor network aiming for variable rate irrigation, real time in field sensing, controlling of a site specific precision linear move irrigation system to maximize the productivity with minimal use of water was developed by Y. Kim . The system described details about the design and instrumentation of variable rate irrigation, wireless sensor network and real time in field sensing and control by using appropriate software. The whole system was developed using five in field sensor stations which collects the data and send it to the base station using global positioning system (GPS) where necessary action was taken for controlling irrigation according to the database available with the system. The system provides a promising low cost wireless solution as well as remote controlling for precision irrigation.

4. Bhagyashree K.Chate, Prof.J.G.Rana et al.[4] have explained about a proposed new innovative irrigation system which comprises of the live streaming of crops using android phones and automatic motor on/off system, this two systems make the irrigation fully automatic. We can capture the live crop images on Wi-Fi. The entire system is monitored and controlled by the power full credit card sized microcomputer called Raspberry Pi. Pi board is powered by Linux operating system.

5. Hui Chang, Nan Zhou, Xiaoguang Zhao, Qimin Cao, Min Tan, Yongbei Zhang et al.[9] have explained that researchers measured soil related parameters such as temperature and humidity. Sensors were placed below the soil which communicates with relay nodes by the use of effective communication protocol providing very low duty cycle and hence increasing the life time of soil monitoring system.

6. The system was developed using microcontroller, universal asynchronous receiver transmitter (UART) interface and sensors while the transmission was done by hourly sampling and buffering the data, transmit it and then checking the status messages. The drawbacks of the system were its cost and deployment of sensor under the soil which causes attenuation of radio frequency (RF) signals.

\section{COMPONENTS USED (Hardware and Software):}

\subsection{Hardware}

\section{Sensors :}

1. Ultrasonic Sensor: An Ultrasonic sensor is a device that can measure the distance to an object by using sound waves. It measures distance by sending out a sound wave at a specific frequency and listening for that sound wave to bounce back. By recording the elapsed time between the sound wave being generated and the sound wave bouncing back, it is possible to calculate the distance between the sonar sensor and the object. Since it is known that sound travels through air at about 344 $\mathrm{m} / \mathrm{s}(1129 \mathrm{ft} / \mathrm{s})$, you can take the time for the sound wave to return and multiply it by 344 meters (or 1129 feet) to find the total round-trip distance of the sound wave. Round-trip means that the sound wave traveled 2 times the distance to the object before it was detected by the sensor; it includes the 'trip' from the sonar sensor to the object AND the 'trip' from the object to the Ultrasonic sensor (after the sound wave bounced off the object). To find the distance to the object, simply divide the round-trip distance in half.

$$
\text { distance }=\frac{\text { speed of sound } \times \text { time taken }}{2}
$$

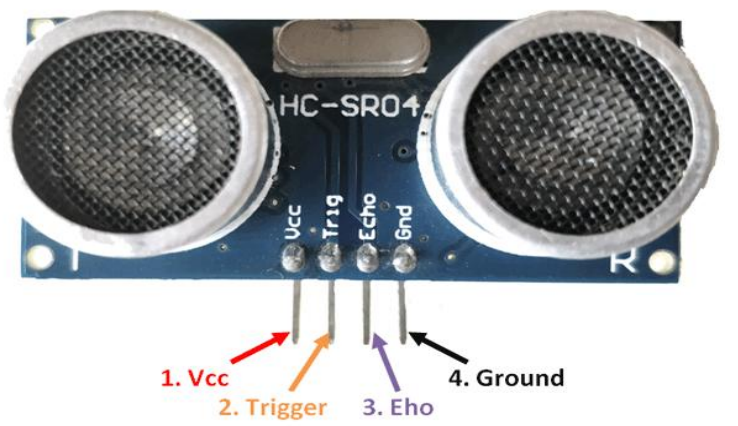

Fig,1

2. Passive Infrared Sensor : A passive infrared sensor (PIR sensor) is an electronic sensor that measures infrared (IR) light radiating from objects in its field of view. It detects a human being moving around within approximately $10 \mathrm{~m}$ from the sensor. This is an average value, as the actual detection range is between $5 \mathrm{~m}$ and $12 \mathrm{~m}$.PIR are fundamentally made of a pyro electric sensor, which can detect levels of infrared radiation. For numerous essential projects or items that need to discover when an individual has left or entered the area. PIR sensors are incredible, they are flat control and minimal effort, have a wide lens range, and are simple to interface with.

Most PIR sensors have a 3-pin connection at the side or bottom. One pin will be ground, another will be signal and the last pin will be power. Power is usually up to $5 \mathrm{~V}$. Sometimes bigger modules don't have direct output and instead just operate a relay which case there is ground, power and the two switch associations. Interfacing PIR with microcontroller is very easy and simple. The PIR acts as a digital output so all you need to do is listening for the pin to flip high or low. The motion can be detected by checking for a high signal on a single $\mathrm{I} / \mathrm{O}$ pin.

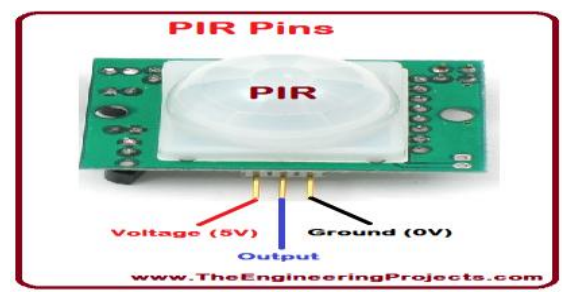

Fig.2

3. Temperature and Humidity Sensor : The DHT11 is a basic, low-cost digital temperature and humidity sensor. It 
gives out digital value and hence there is no need to use conversion algorithm at ADC of the microcontroller and hence we can give its output directly to data pin instead of ADC. It has a capacitive sensor for measuring humidity. This DHT11 Temperature and Humidity Sensor features a calibrated digital signal output with the temperature and humidity sensor capability. It is integrated with a highperformance 8-bit microcontroller. Its technology ensures the high reliability and excellent long-term stability. This sensor includes a resistive element and a sensor for wet NTC temperature measuring devices. It has excellent quality, fast response, anti-interference ability and high performance.

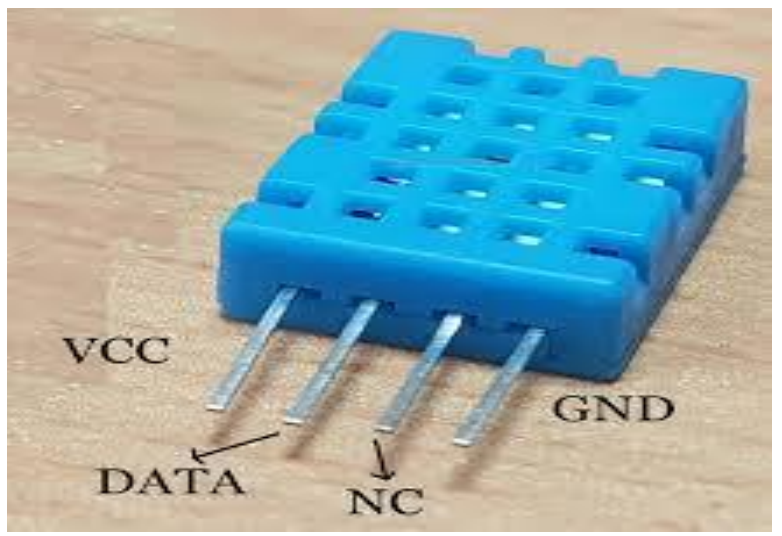

Fig.3

4. Soil Moisture Sensor: Soil Moisture Sensor is used for measuring the moisture in soil and similar materials. The sensor has two large exposed pads which functions as probes for the sensor, together acting as a variable resistor. This sensor detects the moisture level of the soil. When the water level is low in the soil, the analog voltage will be low and this analog voltage keeps increasing as the conductivity between the electrodes in the soil changes. This sensor can be used for watering a flower plant or any other plant requires automation. Technical Specification: $3.3 \mathrm{~V}$ to $5 \mathrm{~V}$; Analog Output; VCC external $3.3 \mathrm{~V}$ to $5 \mathrm{~V}$.

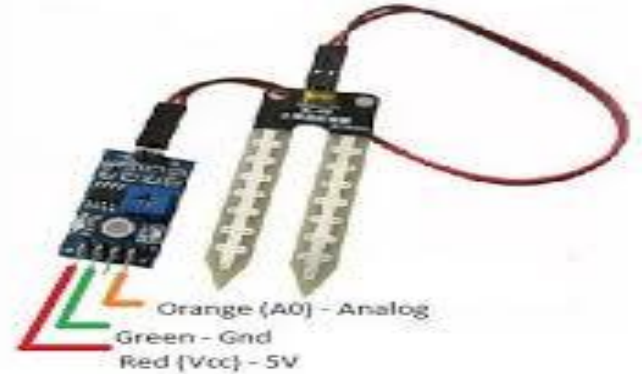

Fig.4

5. Relay: A relay is an electrically operated switch. Many relays use an electromagnet to mechanically operate a switch, but other operating principles are also used, such as solid state relays. Relays are used where it is necessary to control a circuit by a separate low-power signal, or where several circuits must be controlled by one signal.

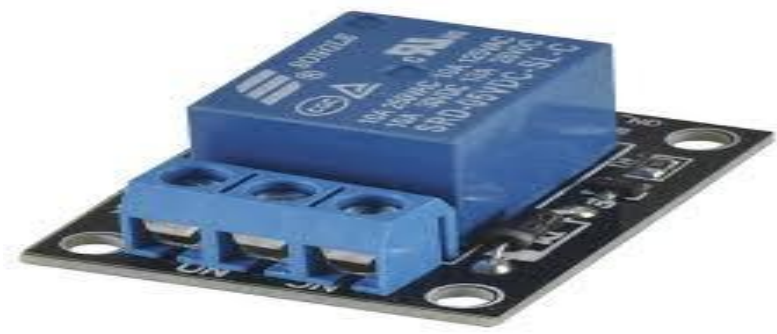

Fig.5

6. Raspberry Pi Touch Display :This 5" touchscreen monitor for Raspberry Pi gives users the ability to create allin-one, integrated projects such as tablets, infotainment systems and embedded projects. The 800 x 480 display connects via an adapter board which handles power and signal conversion. Only two connections to the $\mathrm{Pi}$ are required; power from the Pi's GPIO port and a ribbon cable that connects to the DSI port present on all Raspberry Pi's (except Raspberry Pi Zero and Zero W). Touchscreen drivers with support for 10-finger touch and an on-screen keyboard will be integrated into the latest Raspbian OS for full functionality without a physical keyboard or mouse.

4.1.4 Raspberry Pi Camera: This $5 \mathrm{mp}$ camera module is capable of 1080p video and still images and connects directly to your Raspberry Pi. Connect the included ribbon cable to the CSI (Camera Serial Interface) port on your Raspberry Pi, boot up the latest version of Raspbian and you are good to go! The board itself is tiny, at around $25 \mathrm{~mm} \times 20 \mathrm{~mm} \times 9 \mathrm{~mm}$ and weighing in at just over $3 \mathrm{~g}$, making it perfect for mobile or other applications where size and weight are important. The sensor has a native resolution of 5 megapixel, and has a fixed focus lens onboard. In terms of still images, the camera is capable of 2592 x 1944 pixel static images, and also supports 1080p30, 720p60 and 640x480p60/90 video. Note: This module is only capable of taking pictures and video, not sound. Parameters: Lens: 1/4 5M Aperture: 2.9 Focal Length: 3.29 FOV: 72.4 degrees Hardware connection: 1 soft cable, 90-degree vertical connector, HDMI port next to it.

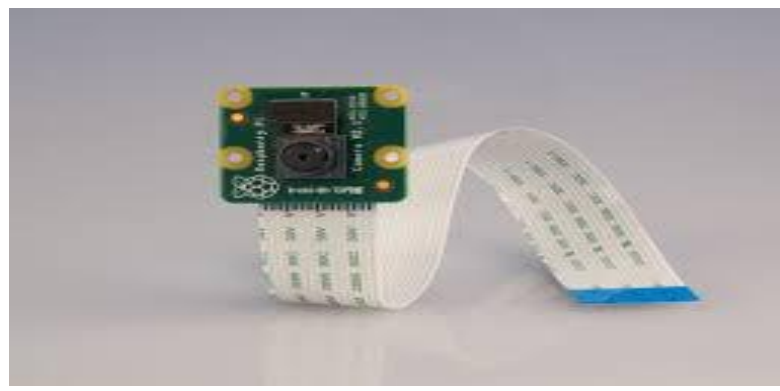

Fig.6

Mini Submersible Pump Motor : This is a low cost, small size Submersible Pump Motor which can be operated from a $2.5 \sim 6 \mathrm{~V}$ power supply. It can take up to 120 liters per hour with very low current consumption of $220 \mathrm{~mA}$. Just connect tube pipe to the motor outlet, submerge it in water and power it. Make sure that the water level is always higher than the motor. Dry run may damage the motor due to heating and it will also produce noise. 


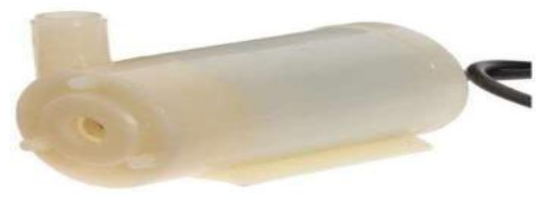

Fig.7

\subsection{Software}

Python: Python is an interpreted high-level programming language for general-purpose programming. Created by Guido van Rossum and first released in 1991, Python has a design philosophy that emphasizes code readability, and a syntax that allows programmers to express concepts in fewer lines of code, notably using significant whitespace. It provides constructs that enable clear programming on both small and large scales.

Thing Speak: Thing Speak is an open source Internet of Things application and API to store and retrieve data from things using the HTTP protocol over the Internet or via a Local Area Network. Thing Speak enables the creation of sensor logging applications, location tracking applications, and a social network of things with status updates. Thing Speak was launched as a service in support of IoT applications

Open CV: Open CV (Open Source Computer Vision Library) is released under a BSD license and hence it's free for both academic and commercial use. It has $\mathrm{C}++$, Python and Java interfaces and supports Windows, Linux, Mac OS, iOS and Android. Open CV was designed for computational efficiency and with a strong focus on real-time applications. Written in optimized $\mathrm{C} / \mathrm{C}++$, the library can take advantage of multi-core processing. Enabled with OpenCL, it can take advantage of the hardware acceleration of the underlying heterogeneous compute platform.

\section{SYSTEM DESIGN}

The block diagram of the proposed system is shown in figure below consists of different sensing unit

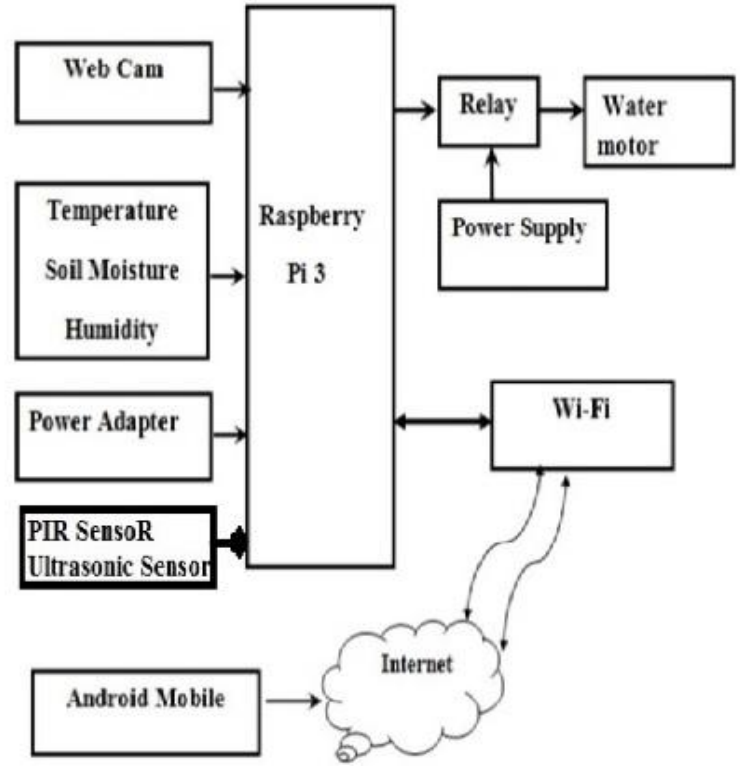

Fig.8

\section{WORKING OF PROJECT}

The proposed system has temperature sensor, humidity sensor and PIR sensors, Ultrasonic sensor. Soil moisture sensor used to obtain the soil moisture level, if the moisture level is low, then the water motor is made on by turning the relay on, and if the soil moisture is normal means, the relay is turned off to off the motor. The PIR sensor is used to detect the object crossing the area, if any movement is detected by the PIR sensor; The web camera records the object and mail it to user. In this entire process, all the activities of the irrigation system is notified to the user as text message by sending an SMS, with the parameters like humidity value, temperature value, Motor status.

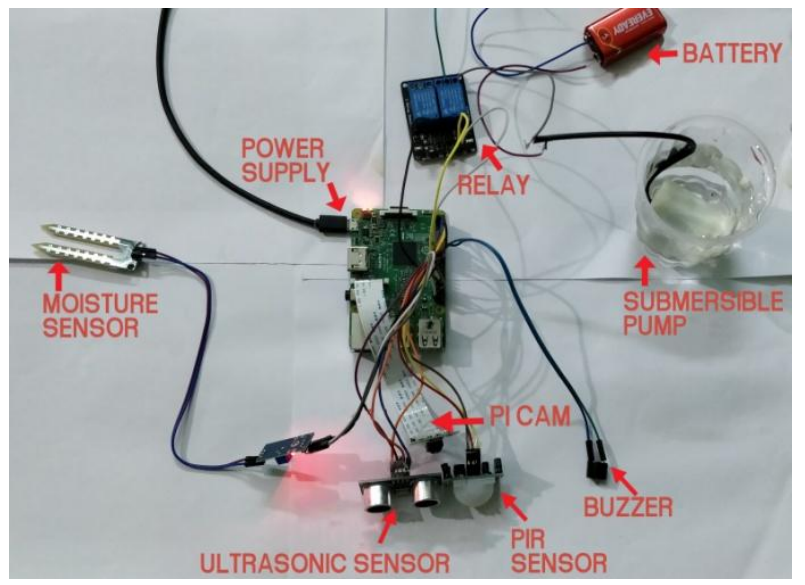

Fig.9

The humidity sensors senses, measures and reports the relative humidity in the air. It therefore measures both moisture and air temperature. Humidity is the ratio. The PIR Sensor is a pyroelectric device that detects motion by measuring changes in the infrared levels emitted by surrounding objects. This motion can be detected by checking for a high signal on a single I/O pin. This can vary with environmental conditions. The sensor is designed to adjust to slowly changing conditions that would happen normally as the day progresses and the environmental conditions change, but responds by toggling its output when sudden changes occur, such as when there is motion. Humidity sensor is placed in the soil to measure the soil wet status. If the soil is wet the value is given to the comparator circuit. In comparator circuit, we set the predefined value using the trim pot, and read the humidity value and compare the two values, if the soil is wet the comparator will give high signal otherwise low signal to the Raspberry pi controller. Depending on the signal the controller will give signal to the relay driver circuit to switch $\mathrm{ON}$ and OFF the water pump. The controller will send the status of the motor (motor on \& motor off). The controller will activate the alarm to intimate that the humidity value is low. And the temperature sensor is used to sense the weather condition. The temperature sensor value is given to the comparator circuit. In comparator circuit we set the predefined value using the trim pot and read the temperature value and compare the two values, if the temperature value is high the comparator will give high signal otherwise low signal to the Raspberry pi controller. When the controller reads high signal, it sends the message to the predefined number using GSM modem. The controller will activate the alarm to intimate that the temperature value is high. And the PIR sensor is used to sense motion of the person or any animal. If any motion is sensed, the PIR sensor gives high signal to the controller. The controller activates the web camera to capture the object, and 
sends to the predefined mail ID, and also sends the message to the predefined number. MAX 232 is used to communicate between GSM and Raspberry Pi controller. In our project we have obtained, a normal farmer can connect this setup to his computer or mobile phones and select the crop type and accordingly he can automatically monitor the irrigation system for the crops along with controlling humidity and temperature in his field. From this it is concluded that we have succeeded in using the technology of "Raspberry pi controller" for an automatic irrigation process using wireless sensor network.

\section{CONCLUSION AND FUTURE WORK}

The smart agriculture system using IOT has been designed and implemented in this paper. The system developed is beneficial and works in cost effective manner. It reduces the water consumption to a great extent. It reduces the maintenance, the power consumption could be reduced by using solar power also. The system can be used in green houses. The System is very useful in areas where water availability is less. The system is also useful in solving the major problems of protecting corps from threatening animal. The productivity of the crops increases and the wastage of crops is very much reduced using this agriculture system. The developed system is more helpful and gives more feasible results.

In the near future, a modern day agriculture farm could be managed as shown in Figure. Based on data from each field, such as the moisture level of soil, temperature of farm, soil condition and conditions of climate where crops are grown, decision models would determine the site specific soil tillage, pre-treatment of the seedbed, and sowing density of the farm. In the growth season, the modern farmer decides about sitespecific application of fertilizers, supported by crop growth models and field measurements, the most important of which is soil coverage of crops in the early growth stage. A spraying machine aided with optical sensors for detection of diseases and weeds is used for the treatment of the crops. We can design a model which aims at eliminating problems like entering animals into the farm by using motion sensor so that it will continuously monitor the output ; if some motion is observed in the farm then the webcam is activated and it captures the image. The captured image is then compared with the saved image sheet and it will be verified whether the image is of the human or animal. If animal is sensed then the buzzer is on with the message from the GSM module to the owner and email is sent. Moisture level is sensed and then the output is monitored. If the deficiency in water is sensed then the data from the weather department was analyzed and if there is a possibility of rain during the next 4-5 hours of the operation then the motor for pumping water is stopped or else the pump was started and then the irrigation system works. Water flow can also be controlled via the analog input of the soil moisture level. If there is a urgent requirement of the irrigation system a reminder via mail is sent to the user for immediate turning on of the dc motor irrigation system remotely via user.

\section{ACKNOWLEDGMENT}

Our thanks to Mr. Ashis Kumar Mishra Lecture in Computer Science and Engineering at College of Engineering and Technology ,Bhubaneswar who has contributed towards development of this thesis.

\section{REFERENCES}

[1]. Smart agriculture using IOT and WSN. Nikesh Gondchawar, Prof. Dr. R. S. Kawitkar on IJIRCCE, ISSN(Online): 2320-9801.

[2]. IOT based Smart Farm Irrigation System using Raspberry Pi. Manoj C Chauhan, Mehul K Vala Associate Professor at department of ECE in Shantilal Shah Government Engineering College, Bhavnagar, Gujarat, India on International Journal Of Applied Research In Science And Engineering Volume 1, Issue 2, June-2016, ISSN:2456-124X.

[3]. Y. Kim, R. Evans and W. Iversen, "Remote Sensing and Control of an Irrigation System Using a Distributed Wireless Sensor Network", IEEE Transactions on Instrumentation and Measurement, pp. 1379-1387, 2008.

[4]. Smart Irrigation System using Raspberry pi . Bhagyashree K.Chate, Prof.J.G.Rana in International Research Journal of Engineering and Technology ,IRJET having e-ISSN: 2395-0056.

[5]. Raspberry pi using IR THERMAL CAMERA in agriculture farm for SMART IRRIGATION SYSTEM on International Research Journal of Engineering and Technology (IRJET) p- ISSN: 2395-0072.

[6]. GSM based automated irrigation control using raingun irrigation system,R.Sureshvol 3 , issue $2^{\text {nd }}$ feb2014,IJRCCE.

[7]. Sensor data collection and irrigation control on vegetable crop using smart phone and wireless network for smart farm: Kaewmard N, Saiyod S, 2014 IEEE.

[8]. Smart farming system using sensor for agriculture task automation: Dwarkani M C, Ram R G, Jagannatham S.

[9]. Hui Chang, Nan Zhou, Xiaoguang Zhao, Qimin Cao, Min Tan,Yongbei Zhang. ("A New Agriculture Monitoring System Based on WSN" The State Key Lab of Management and Control for Complex System Institute of Automation, Chinese Academy of Sciences, Beijing, China and HaiNan State Farms Academy of Sciences, Haikou, China 978-1-4799-2186-7114 (02014 IEEE).

[10]. Stefanos A. Nikolidakis, Dionisis Kandris,Dimitrios D. Vergadoschristos Douligeris A"Energy Efficient Automated Control Of Irrigation In Agriculture By Using Wireless Sensor Networks, Computers And Electronics In Agriculture "0168- 1699/ 2015 Elsevier B.V.

[11]. Venkata Naga Rohit Gunturi, "Micro Controller Based Automatic Plant Irrigation System" International Journal of Advancements in Research \& Technology, Volume 2, Issue- 4, April-2013.

[12]. R.Hussain, J.Sehgal, A.Gangwar, M.Riyag"Control of irrigation automatically by using wireless sensor network" International journal of soft computing and engineering, vol.3, issue 1, march 2013, pp.48t 324-328.

[13]. G. Yuan, Y. Luo, X. Sun, and D. Tang, "Evaluation of a crop water stress index for detecting water stress in winter wheat in the North China Plain,"Agricult. Water Manag., vol. 64, no. 1, pp. 29-40, Jan. 2004.

[14]. Chandan Kumar Sahu, Dept. of Electronics and communication engineering. Sambalpur University 
Institute of Information Technology, Sambalpur(768019), INDIA chandan.sahu@suiit.ac.in Pramitee Behera, Dept. of Electronics and communication engineering. Sambalpur University Institute of Information Technology Sambalpur(768019), INDIA pramitee.behera@suiit.ac.in, A Low Cost Smart Irrigation Control System, IEEE SPONSORED 2ND INTERNATIONAL CONFERENCE ON ELECTRONICS AND COMMUNICATION SYSTEM (ICECS 2015) 978-1-4788-7225-8/15/\$31.00 @ 2015 IEEE.

[15]. Nikhil Agrawal Engineering Manager, Siemens, Noida nikhil.pa@gmail.com, Smita Singhal ASET, Amity
University, Noida singhal.smita@gmail.com , Smart Drip Irrigation System using Raspberry pi and Arduino, International Conference on Computing, Communication and Automation (ICCCA2015) ISBN:978-1-4799-8890-7/15/\$31.00 @2015 IEEE .

[16]. Hui Chang, Nan Zhou, Xiaoguang Zhao, Qimin Cao, Min Tan,Yongbei Zhang. "A New Agriculture Monitoring System Based on WSN" The State Key Lab of Management and Control for Complex System Institute of Automation, Chinese Academy of Sciences, Beijing, China and HaiNan State Farms Academy of Sciences, Haikou, China 978-1-4799-2186-7114 @201 4 IEEE . 